\title{
Elevated Levels of StAR-Related Lipid Transfer Protein 3 Alter Cholesterol Balance and Adhesiveness of Breast Cancer Cells
}

\section{Potential Mechanisms Contributing to Progression of HER2-Positive Breast Cancers}

Boris Vassilev, ${ }^{*}$ Harri Sihto, ${ }^{\dagger}$ Shiqian Li, ${ }^{\star \ddagger}$ Maarit Hölttä-Vuori, ${ }^{* \ddagger}$ Jaakko Ilola, ${ }^{*}$ Johan Lundin, ${ }^{\S}$ Jorma Isola, Pirkko-Liisa Kellokumpu-Lehtinen, "Heikki Joensuu, and Elina Ikonen ${ }^{* \ddagger}$

From the Faculty of Medicine, ${ }^{*}$ Department of Anatomy, and the Laboratory of Molecular Oncology, ${ }^{\dagger}$ Translational Cancer Biology Program, University of Helsinki, Helsinki; the Minerva Foundation Institute for Medical Research, ${ }^{\ddagger}$ Biomedicum $2 U$, Helsinki; the Institute for Molecular Medicine Finland, ${ }^{\S}$ University of Helsinki, Biomedicum Helsinki 2U, Helsinki; the Institute of Medical Technology, "University of Tampere and Tampere University Central Hospital, Tampere; and the Department of Oncology," Helsinki University Central Hospital, Helsinki, Finland

\author{
Accepted for publication \\ December 9, 2014. \\ Address correspondence to Elina \\ Ikonen, M.D., Ph.D., Faculty of \\ Medicine, Department of \\ Anatomy, University of Helsinki, \\ Haartmaninkatu 8, FI-00290 \\ Helsinki, Finland. E-mail: elina. \\ ikonen@helsinki.fi.
}

\begin{abstract}
The STARD3 gene belongs to the minimal amplicon in HER2-positive breast cancers and encodes a cholesterol-binding membrane protein. To study how elevated StAR-related lipid transfer protein 3 (StARD3) expression affects breast cancer cells, we generated MCF-7 cells stably overexpressing StARD3-green fluorescent protein. We found that StARD3-overexpressing cells exhibited nonadherent morphological features, had increased Src levels, and had altered cholesterol balance, as evidenced by elevated mRNA levels of the cholesterol biosynthesis rate-limiting enzyme 3hydroxy-3-methylglutaryl-coenzyme A reductase, and increased plasma membrane cholesterol content. On removal of serum and insulin from the culture medium, the morphological characteristics of the StARD3-overexpressing cells changed, the cells became adherent, and they developed enlarged focal adhesions. Under these conditions, the StARD3-overexpressing cells maintained elevated Src and plasma membrane cholesterol content and showed increased phosphorylation of focal adhesion kinase. In two Finnish nationwide patient cohorts, approximately $10 \%(212 / 2220)$ breast cancers exhibited high StARD3 protein levels, which was strongly associated with HER2 amplification; several factors related to poor disease outcome and poor breast cancer-specific survival. In addition, high StARD3 levels in breast cancers were associated with elevated 3-hydroxy-3-methylglutaryl-coenzyme A reductase mRNA levels and anti-Src-Tyr416 immunoreactivity. These results provide evidence that StARD3 overexpression results in increased cholesterol biosynthesis and Src kinase activity in breast cancer cells and suggest that elevated StARD3 expression may contribute to breast cancer aggressiveness by increasing membrane cholesterol and enhancing oncogenic signaling. (Am J Pathol 2015, 185: 987-1000; http:// dx.doi.org/10.1016/j.ajpath.2014.12.018)
\end{abstract}

The genomic region in chromosome 17 containing the HER2 $(E R B B 2)$ oncogene $(17 \mathrm{q} 12-\mathrm{q} 21)$ is frequently amplified in cancers. This region contains multiple genes coamplified with HER2. ${ }^{1,2}$ Several of these genes, including STARD3, have been suggested to functionally contribute to the proliferation of
Supported by the Helsinki Biomedical Graduate Programme (B.V.), Academy of Finland grants 131489, 263841, and 272130 (E.I.), Institute for Molecular Medicine Finland national network funding (E.I.), and Sigrid Juselius Foundation (E.I.).

Disclosures: None declared. 
HER2-positive cells, on the basis of RNA interference studies. ${ }^{3,4}$ The receptor tyrosine-protein kinase erbB-2 (ErbB2) protein is a cell surface transmembrane tyrosine kinase that forms long-lived, potent heterodimers with other receptors of the epidermal growth factor receptor family, and acts as a networking receptor that mediates growth, survival, migration, and proliferation signals to cancer cells. ${ }^{5,6}$ A monoclonal antibody directed against the extracellular domain of ErbB2, trastuzumab, forms the basis for the treatment of HER2-positive breast cancers. However, more than half of HER2-positive cancers either are or become resistant to the drug, implying that other genes, such as those coamplified with HER2, may contribute to the aggressive behavior of these tumors. ${ }^{3,4,7}$

The StAR-related lipid transfer protein 3 (StARD3; alias MLN64 for metastatic lymph node 64) transcript was originally identified as being highly expressed in 14 of 93 invasive breast carcinomas that also expressed high HER2 mRNA levels. ${ }^{8}$ The STARD3 transcript encodes a ubiquitously expressed protein that localizes to the late endosomal organelles (LEs) ${ }^{9}$ and binds cholesterol. ${ }^{10}$ Its N-terminal domain with four transmembrane spans targets the protein to the LE membrane, ${ }^{9,11}$ and its C-terminal START domain harbors a hydrophobic cholesterol-binding pocket that projects toward the cytoplasm. ${ }^{10}$ The $\mathrm{N}$-terminal domain is also capable of interacting with cholesterol. ${ }^{12}$ Despite considerable interest in the StARD3 protein, its precise function is not well understood. Targeted disruption of the START domain causes only modest alterations in whole body cholesterol metabolism in mice ${ }^{13}$ However, there are several indications that cholesterol binding or transfer by StARD3 affects cellular functions. The cholesterol-binding activity of StARD3 is involved in controlling the association of LEs with the actin cytoskeleton and the actin-dependent movement of LEs. ${ }^{14}$ The protein can also facilitate the transfer of cholesterol between LEs and other membrane compartments, such as mitochondria ${ }^{15}$ and the plasma membrane. ${ }^{11}$ The nascent StARD3 protein is transported to the LEs via the plasma membrane. ${ }^{16}$

Recent work suggests that the coamplification of STARD3 with $E R B B 2$ might play a role in breast cancer progression. First, StARD3 overexpression, as measured by quantitative real-time PCR, increased the prognostic power of ErbB2 overexpression for disease-free survival of breast cancer patients. ${ }^{17}$ Second, knockdown of StARD3 by siRNA in HER2-positive breast cancer cell lines (both trastuzumabresponding cell lines BT474 and SKBR3 and nonresponding lines JIMT1 and KPL4) led to induction of apoptosis, suggesting that StARD3 is required for the growth and survival of these cells. ${ }^{4}$ However, the mechanisms by which StARD3 may mediate these effects are not known.

To learn whether increased StARD3 protein amounts can alter the characteristics of cancer cells independently of HER2 amplification, we investigated the effects of StARD3 overexpression in a well-studied model of HER2-negative human breast cancer MCF-7 cells. We provide evidence that the elevated expression of StARD3 protein is accompanied by significant morphological and biochemical alterations in these cells, including altered membrane cholesterol content and enhanced signaling related to cell adhesion. We also raised a polyclonal StARD3-specific antibody and demonstrate high StARD3 immunoreactivity, coinciding with HER2 amplification, in 1 of 10 human breast tumors in two cohorts of $>2000$ patients. High StARD3 levels also associate with indicators of altered cholesterol metabolism and signaling in these tumors. Together, our data suggest that, in highly StARD3-expressing breast cancer cells, the cholesterol-mobilizing and membranealtering properties of the protein might exaggerate oncogenic signaling via membrane-associated kinases and thereby contribute to breast cancer aggressiveness.

\section{Materials and Methods}

\section{Antibodies}

To raise anti-StARD3 antibodies, the START domain of the StARD3 protein (corresponding to amino acids 230 to 445) was expressed as a His6-glutathione $S$-transferase fusion protein in Escherichia coli JM109(DE3) and purified from cell debris on preparative SDS-polyacrylamide gels. New Zealand white rabbits were immunized with the purified protein. The polyclonal antiserum was affinity purified using the glutathione $S$-transferase-fusion protein and used at $1: 10$ dilution for immunofluorescence staining and at 1:50 to 1:200 dilution for immunoblotting. Anti-ErbB2 mouse monoclonal antibody (3B5; ab16901) was purchased from Abcam (Cambridge, UK), Lamp-1 H43A-c mouse monoclonal antibody was from the Developmental Studies Hybridoma Bank (University of Iowa, Iowa City), sterol regulatory element-binding protein 2 mouse monoclonal antibody (557037) was from BD Biosciences (San Jose, CA), phospho-Src (Tyr416) rabbit polyclonal antibody (number 2101) was from Cell Signaling Technology (Danvers, MA), anti-focal adhesion kinase (pp125FAK) rabbit antibody (F2918) was from Sigma-Aldrich (St. Louis, MO), and FAK (pY397) purified mouse antibody (611807) was from BD Transduction Laboratories (San Jose, CA).

\section{Cell Culture}

MCF-7 cells were cultured in Eagle's minimum essential medium from Lonza (Verviers, Belgium), supplemented with $10 \%$ fetal bovine serum (10270-106) from Life Technologies (Grand Island, NY), $0.5 \mathrm{mmol} / \mathrm{L}$ L-glutamine (25030-024) from Life Technologies, $0.01 \mathrm{mg} / \mathrm{mL}$ insulin (human, rDNA) from Novo Nordisk (Bagsværd, Denmark), MEM nonessential amino acids (M7145) from Sigma-Aldrich, and $100 \mathrm{IU} / \mathrm{mL}$ penicillin and $100 \mu \mathrm{g} / \mathrm{mL}$ streptomycin (DE17-602E) from Lonza. Flasks and dishes were purchased from Nunc (Roskilde, Denmark), and 12- and 6-well dishes were from Falcon (Becton-Dickinson Labware, Franklin Lakes, NJ).

For the control cell line, a pEGFP-C1 empty plasmid from BD Biosciences Clontech (Palo Alto, CA) was used. The plasmid encoding green fluorescent protein (GFP)-fused full-length 

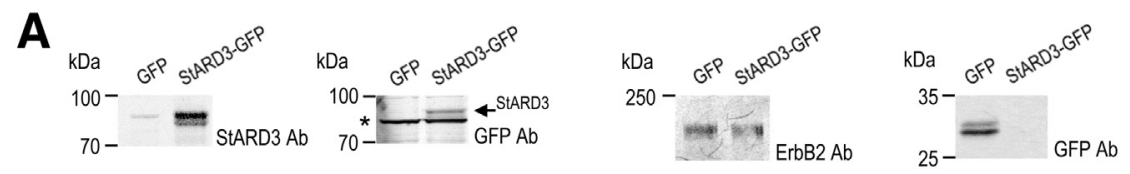

B

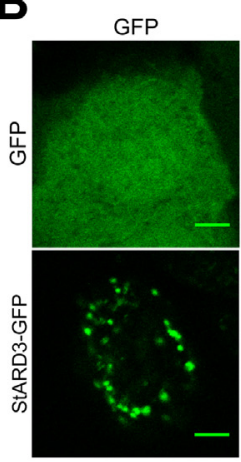

Lamp-1
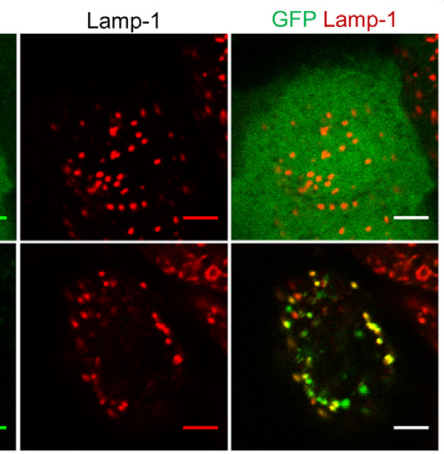

C
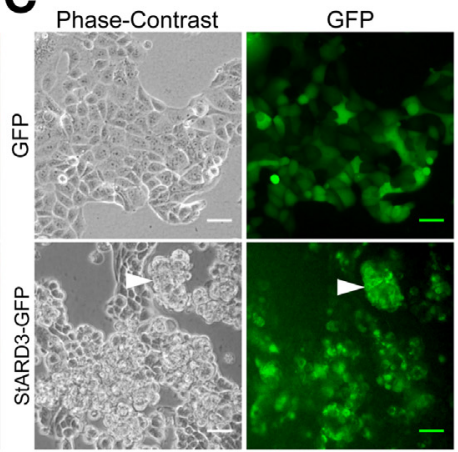

D

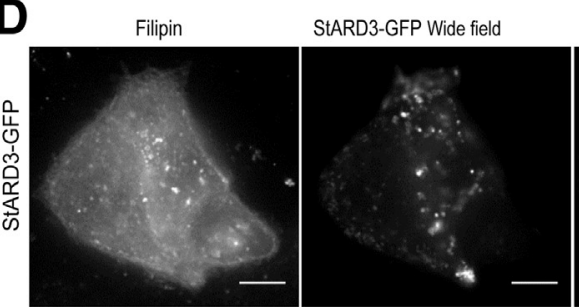

StARD3-GFP TIRF

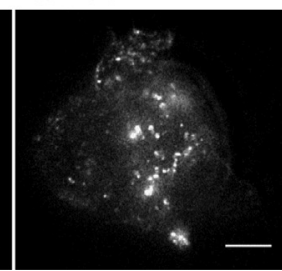

TIRF / Wide field

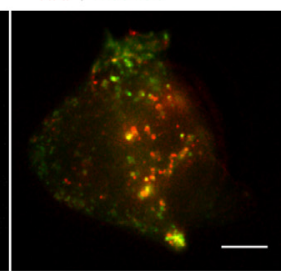

Figure 1 StAR-related lipid transfer protein 3green fluorescent protein (StARD3-GFP) overexpression in breast cancer MCF-7 cells results in clustered growth of rounded-up cells without increasing ErbB2 levels. A: Immunoblots of cell lysates from control GFP and StARD3-GFP-expressing MCF-7 cell lines. Equal amounts of total protein were separated by SDS-PAGE, transferred by Western blot analysis, and detected by immunostaining with the indicated antibodies (Abs). The anti-GFP antibody detects a cross-reactive band (asterisk) close to the StARD3 band (arrow). B: Fluorescence micrographs of GFP and StARD3-GFP cells. Cells were fixed with paraformaldehyde, immunostained with anti-Lamp-1 antibodies, and imaged with a confocal microscope at the focal plane with most prominent Lamp-1 staining. C: Micrographs of live cells. Phase-contrast and widefield fluorescence images. Arrowheads indicate a strongly StARD3-GFP-expressing, poorly adherent cell cluster. D: Wide-field fluorescence and total internal reflection fluorescence (TIRF) micrograph of a StARD3-GFP-expressing cell. Cells were fixed with PFA, stained with filipin, and imaged with a TIRF microscope for plasma membrane visualization. Scale bars: $5 \mu \mathrm{m}$ (B); $20 \mu \mathrm{m}$ (C); $10 \mu \mathrm{m}$ (D).
StARD3 has been described. ${ }^{14}$ To establish a StARD3overexpressing cell line, cells were seeded at $1.25 \times 10^{5}$ cells per well on 24-well plates and transfected with $1 \mu \mathrm{g}$ plasmid with Lipofectamine-LTX (Life Technologies) at 1:3 ratio, according to the manufacturer's protocol. After 48 hours, cells were passaged into medium containing 0.4 or $0.75 \mathrm{mg} / \mathrm{mL}$ geneticin G-418 sulfate (Life Technologies) and selected for 3 weeks before single-cell cloning. Expression efficiency of single clones was screened by fluorescence microscopy. For the StARD3-GFP-overexpressing cell line, single-cell cloning was performed a second time to obtain higher StARD3-GFP expression. Both stable cell lines were maintained with $0.2 \mathrm{mg} / \mathrm{mL}$ geneticin G-418 sulfate. The GFP control cell line was split twice a week at 1:3 and 1:6 ratios. The StARD3-GFP cell line was split once a week at 1:3 and 1:6 ratios, and medium was changed once between splitting. To inhibit cholesterol synthesis, cells were treated for 24 hours with $10 \mu \mathrm{mol} / \mathrm{L}$ lovastatin (Merck \& Co, White House Station, NJ) dissolved in dimethyl sulfoxide (SigmaAldrich). Control samples were treated with dimethyl sulfoxide only.

\section{Immunoblotting}

Cell lysates for Western blot analysis were collected in denaturing lysis buffer containing $5 \mathrm{mmol} / \mathrm{L}$ EDTA and $1 \%$ SDS in phosphate-buffered saline, with added protease inhibitors [chymostatin, leupeptin, antipain, and pepstatin A (all from Sigma-Aldrich) at a final concentration of $25 \mu \mathrm{g} / \mu \mathrm{L}$ each] and phosphatase inhibitors [activated Na-ortho-vanadate (Sigma-Aldrich) at $2 \mathrm{mmol} / \mathrm{L}$ final concentration and $\mathrm{NaF}$
(Sigma-Aldrich) at $25 \mathrm{mmol} / \mathrm{L}$ final concentration]. For Western blot analysis, comparable amounts of cells were collected in denaturing lysis buffer containing protease inhibitors and chymostatin, leupeptin, antipain, and pepstatin A. Protein concentration was determined with the DC Protein Assay from Bio-Rad Laboratories (Hercules, CA). Proteins (20 $\mu \mathrm{g}$ of protein per lane) were separated by SDS-PAGE, transferred to a nitrocellulose membrane, immunoblotted and detected with an Odyssey infrared scanner from LI-COR (Lincoln, NE) or, alternatively, with Amersham ECL Western Blotting Detection Reagents (RPN2106) on Amersham Hyperfilm ECL (28-9068-36) from GE Healthcare Limited (Buckinghamshire, UK). The resulting images were quantified with ImageJ software version 1.48 (NIH, Bethesda, MD). ${ }^{18}$

\section{Fluorescence Staining and Imaging of Cells}

For imaging focal adhesions (FAs), cells were fixed and permeabilized with methanol for 5 minutes at $-20^{\circ} \mathrm{C}$. Otherwise, cells on coverslips were fixed with $4 \%$ paraformaldehyde for 20 minutes at room temperature, and autofluorescence was quenched in $50 \mathrm{mmol} / \mathrm{L}$ ammonium chloride for 15 minutes at room temperature. For filipin staining and permeabilization, the cells were fixed and stained with $0.05 \%$ filipin (F9765) from Sigma-Aldrich in $10 \%$ fetal bovine serum in phosphatebuffered saline for 30 minutes at $37^{\circ} \mathrm{C}$. Otherwise, cells were permeabilized with $0.1 \%$ Triton X-100 (T8787) from SigmaAldrich in phosphate-buffered saline for 5 minutes at room temperature and blocked with $10 \%$ fetal bovine serum for 30 minutes at $37^{\circ} \mathrm{C}$. Primary and secondary antibody incubation was performed sequentially. Red/green fluorescence images 


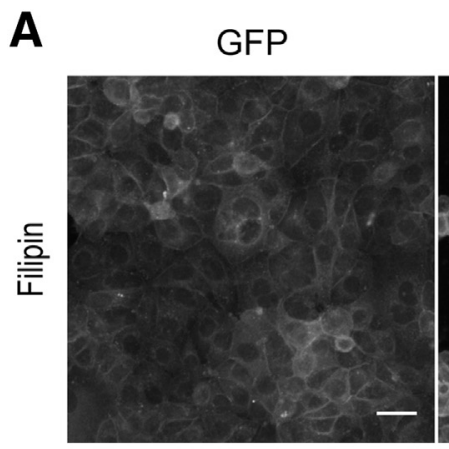

D

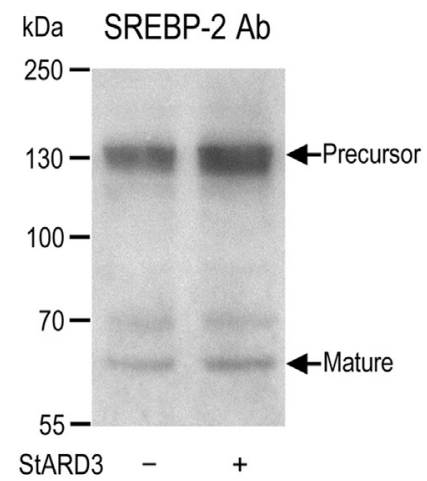

StARD3-GFP

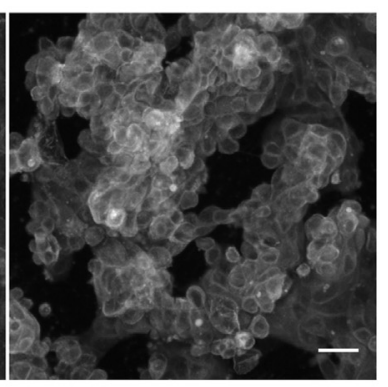

E

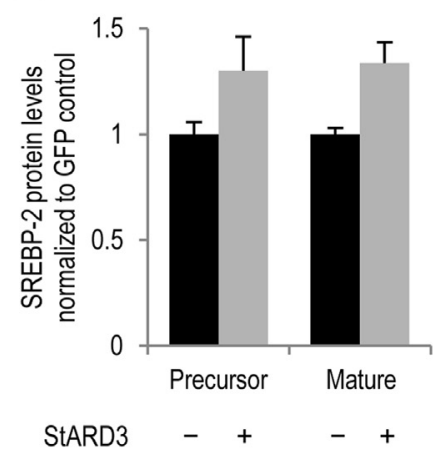

C

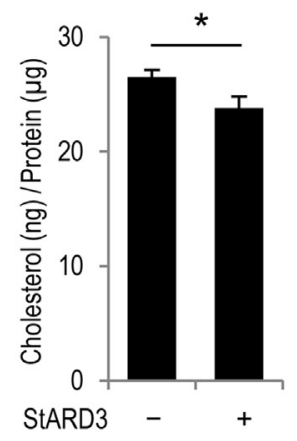

$\mathbf{F}$

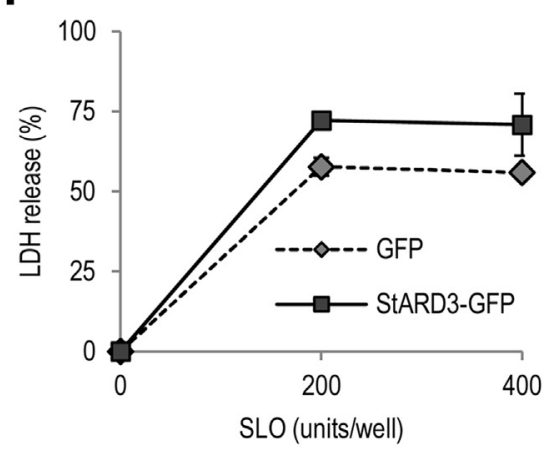

Figure 2 StAR-related lipid transfer protein 3-green fluorescent protein (StARD3-GFP) overexpression results in increased plasma membrane cholesterol and 3hydroxy-3-methylglutaryl-coenzyme A reductase (HMGR) transcript levels. A: Filipin staining. Cells grown in full medium were fixed with paraformaldehye, stained with filipin, and imaged with a wide-field fluorescence microscope. B: Determination of cholesterol levels. Lipids were extracted, and the amount of free cholesterol was analyzed biochemically. C: Analysis of HMGR transcript levels. Total RNA extracted from cells was reverse transcribed, and HMGR cDNA was quantified by real-time RT-PCR. D: Immunoblots of cell lysates. Equal protein amounts were separated by SDS-PAGE, transferred by Western blot analysis, and detected by immunostaining with sterol regulatory element-binding protein 2 (SREBP-2) antibody. The approximately 130-kDa precursor and the approximately 66-kDa mature form are indicated. E: Quantification of SREBP-2 precursor and mature form levels by densitometry. $P=0.007$ versus GFP control cell line for SREBP-2 mature form. F: Sensitivity of cells to streptolysin 0 (SLO). Resistance of the plasma membrane to SLO was measured as the fraction of total cellular lactate dehydrogenase (LDH) released to the medium after 30 minutes of treatment at $37^{\circ} \mathrm{C}$. The measurements at $0 \mathrm{U}$ per well SLO were lower than the sensitivity of the assay and set to 0 . Data represent means \pm SEM for content of cholesterol $(\mathrm{ng})$ per protein $(\mu \mathrm{g})(\mathbf{B})$; HMGR transcript levels normalized to control (MCF-7-GFP cells) (C); seven replicates per condition from two independent experiments normalized to control GFP cells (E); two independent experiments $(\mathbf{F}) . n=6$ from two independent experiments $(\mathbf{B}) ; n=7$ from three independent experiments (C). ${ }^{*} P<0.05$ versus GFP control cell line (Student's $t$-test). Scale bar $=20 \mu \mathrm{m}(\mathbf{A})$.

were acquired with an Olympus AX70 microscope from Olympus Corporation (Hamburg, Germany) equipped with an Olympus DP71 charge-coupled device camera using $10 \times$ or $20 \times$ air objectives. Filipin (UV) images were acquired with the same microscope and with an inverted Olympus microscope with a Polychrome IV monochromator by TILL Photonics (Gräfelfing, Germany) equipped with a TILL IMAGO-QE camera using a $10 \times$ air objective. Confocal images were acquired with a Leica TCS SP2 AOBS confocal microscope from Leica Microsystems (Mannheim, Germany) with a $63 \times$ oil immersion objective. Total internal reflection fluorescence microscopy images were acquired with a Nikon Eclipse Ti-E inverted microscope (Nikon Corporation, Kanagawa, Japan) equipped with an argon laser and a $100 \times$ oil immersion objective (numerical aperture, 1.49). The corresponding widefield images of filipin and StARD3-GFP fluorescence were taken at a focal plane $1 \mu \mathrm{m}$ above the total internal reflection fluorescence. Phase-contrast and green fluorescent images of live cells and fixed cells were acquired with a Zeiss AxioVert 200 microscope from Carl Zeiss Jena (Jena, Germany) equipped with a Zeiss AxioCam HRc color camera using $10 \times$ air objective. All quantifications were done with ImageJ software version $1.48 .^{18}$

\section{Lipid Extraction and Quantification}

Lipids were extracted using the Bligh and Dyer method. ${ }^{19}$ Free cholesterol was resolved on a high performance thin layer chromatography plate using hexane/diethyl ether/acetic acid (80:20:1) as the mobile phase, visualized by charring, and quantified as described previously. ${ }^{20}$

\section{SL0 Permeabilization}

Streptolysin O (SLO) from Streptococcus pyogenes (S0149), $\beta$-nicotinamide adenine dinucleotide, reduced dipotassium salt (N4505), and sodium pyruvate (P2256) were purchased from Sigma-Aldrich. SLO was dissolved in deionized water to $50 \mathrm{U} / \mu \mathrm{L}$, and stored at $-70^{\circ} \mathrm{C}$. SLO activation and membrane permeabilization were performed 

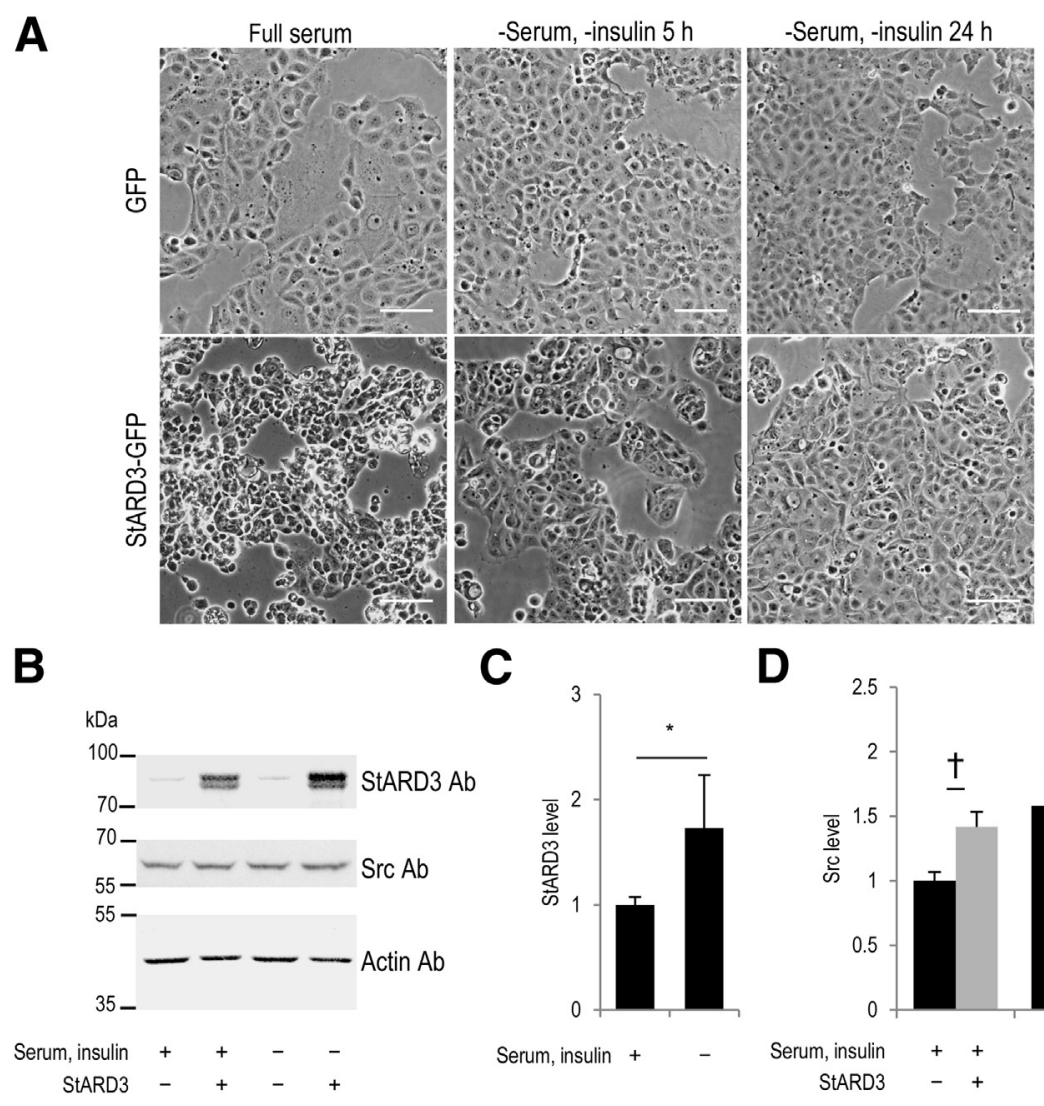

C
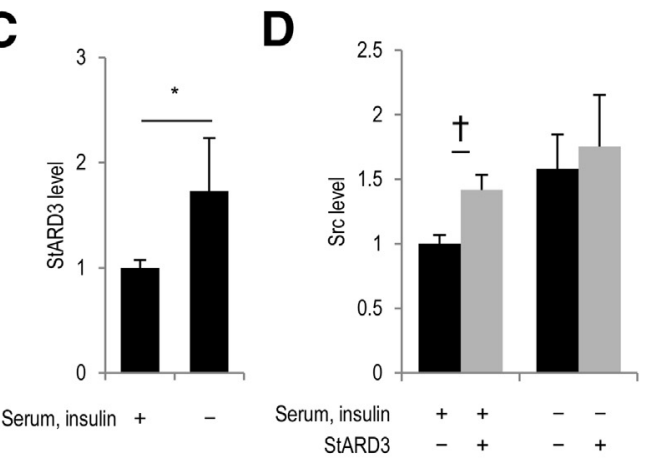

Figure 3 Removal of serum and insulin from the growth medium increases the adherence of StAR-related lipid transfer protein 3 (StARD3)overexpressing cells. A: Micrographs of cells grown in the presence or absence of serum and insulin. Phase-contrast images of green fluorescent protein (GFP) and StARD3-GFP cells deprived of serum and insulin for 0,5 , or 24 hours and fixed with paraformaldehyde. B: Immunoblots of cell lysates. Representative blots of GFP (StARD3-) and StARD3-GFP (StARD3 + ) cells grown in full medium (+serum, +insulin) or deprived of serum and insulin (-serum, -insulin) for 24 hours. Equal amounts of total protein were separated by SDSPAGE, transferred by Western blot analysis, and detected with anti-StARD3, anti-Src, or anti-actin antibodies (Abs). C: Quantification of StARD3 levels by densitometry. D: Quantification of SrC levels by densitometry. Data represent means \pm SEM for six replicates per condition from three independent experiments normalized to control (GFP cells, +serum, +insulin) (C) or six replicates per condition from three independent experiments normalized to control (GFP control cells, +serum, +insulin) (D). ${ }^{*} P<0.05$ cells in -serum, -insulin versus complete medium (Student's $t$-test); ${ }^{\dagger} P<0.05$ versus GFP control cell line (Student's $t$-test). Scale bar $=40 \mu \mathrm{m}$. as previously described. ${ }^{21}$ Briefly, nonconfluent cells in 12well plates were washed and incubated in potassium acetate buffer $[115 \mathrm{mmol} / \mathrm{L}$ potassium acetate (Sigma-Aldrich) and $25 \mathrm{mmol} / \mathrm{L}$ HEPES (Amresco, Solon, $\mathrm{OH}$ )] for 30 minutes at $37^{\circ} \mathrm{C}$. SLO was activated by adding dithiothreitol to a final concentration of $10 \mathrm{mmol} / \mathrm{L}$ and incubating for 30 minutes at $37^{\circ} \mathrm{C}$. The activated SLO was immediately added to the cells at the indicated amounts and incubated for 30 minutes at $37^{\circ} \mathrm{C}$. The medium was collected, and the cells were lysed with $0.1 \%$ Triton X-100 (Sigma-Aldrich) in potassium acetate buffer. The decrease in absorbance at 340 $\mathrm{nm}$, resulting from the oxidation of NADH on addition of pyruvate to medium and cell lysate, was measured over 1 minute. The fraction of lactate dehydrogenase (LDH) released to the medium after membrane permeabilization was calculated as the ratio of LDH in the medium/sum of LDH in the medium and in the cell lysate in each sample.

\section{Patient Samples}

A Finnish nationwide population-based breast cancer series of primary tumors, FinProg, was collected as described previously. ${ }^{22}$ In brief, women diagnosed with breast cancer in five well-defined areas of Finland from 1991 to 1992 were identified, and individual clinicopathological data were collected from the hospital case records and tumor tissue samples from the archives of the pathological departments. Those patients whose StARD3 staining was noninformative were excluded from the study. In total, 1325 of 2930 patients were included in the study. In another study, FinHer, ${ }^{23}$ axillary node-positive or high-risk node-negative samples were collected from 2000 to 2003 from women who had undergone a breast surgery with auxiliary node dissection or sentinel node biopsy for invasive breast carcinoma. Herein, we included a total of 895 patients of the 1010 who participated in the study (approximately $40 \%$ of the eligible women in Finland who received a diagnosis of breast cancer within this period). Permission to use formalin-fixed, paraffinembedded tissues for research purposes from the FinProg study was provided as per local legislation by the Ministry of Social Affairs and Health, Finland (permission 123/08/97). An ethics committee at the Helsinki University Central Hospital (Helsinki, Finland) approved the FinHer study and the current study (permission HUS 125/13/03/02/2014). Study participants provided written informed consent before tissue sample collection or initiation of the study treatments.

Tissue microarrays were constructed as described earlier. ${ }^{22}$ Tissue microarray sections ( $5 \mu \mathrm{m}$ thick) were cut on SuperFrost+ slides from Menzel-Gläser (Braunschweig, Germany), deparaffinized in xylene, and rehydrated through a decreasing alcohol gradient. Endogenous peroxidase activity was blocked with $1 \%$ hydrogen peroxide. Antigen retrieval for Src-Tyr416 was performed in $10 \mathrm{mmol} / \mathrm{L}$ sodium citrate $(\mathrm{pH}$ 6.0) using an autoclave at $120^{\circ} \mathrm{C}$ for 2 minutes. Antigen retrieval for StARD3 was performed in Tris-EDTA $(10 \mathrm{mmol} / \mathrm{L}$ Tris-Base and $1 \mathrm{mmol} / \mathrm{L}$ EDTA, pH 9.0) in a water bath at $98^{\circ} \mathrm{C}$ for 20 
A

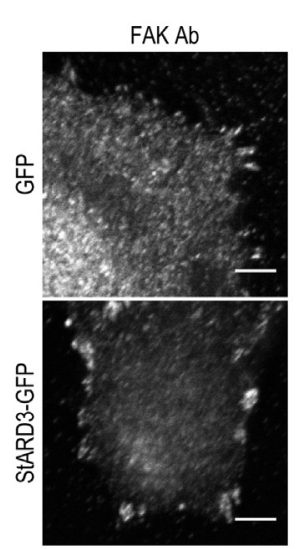

B

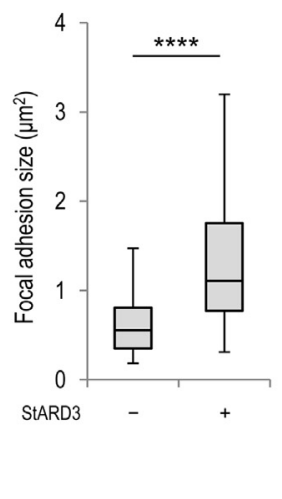

C

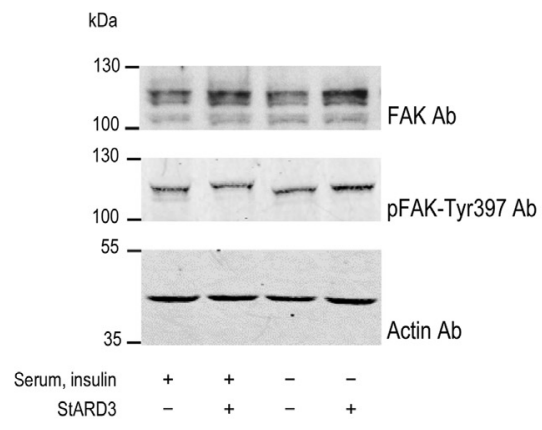

D
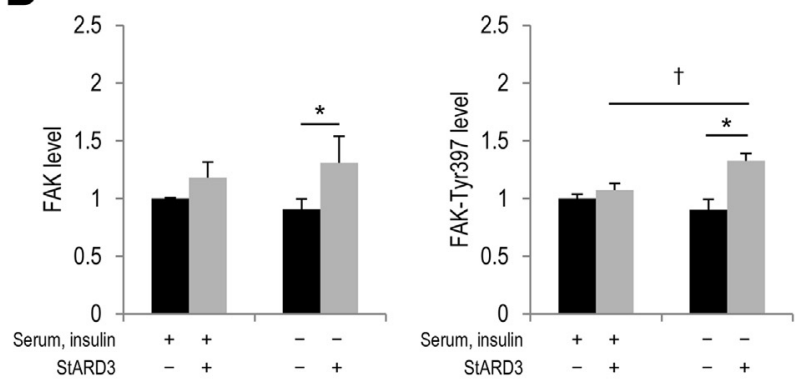

Figure 4 StAR-related lipid transfer protein 3 (StARD3) overexpression causes an increase in focal adhesion size and increased phosphorylation of focal adhesion kinase (FAK) in the absence of serum and insulin. A: FAK staining in the absence of serum and insulin. Cells were deprived of serum and insulin for 24 hours, fixed with methanol, immunostained with anti-FAK antibodies (Abs), and imaged with a confocal microscope at the basal plane, which has the most prominent FAK staining. B: Quantification of focal adhesion size from fluorescence micrographs. The size of FAK-stained patches was analyzed from micrographs (10 cells for each condition). Box plot shows focal adhesion size in $\mu \mathrm{m}^{2}$. Green fluorescent protein (GFP) cells: median $=0.55, n=$ 83; StARD3-GFP cells: median $=1.10, n=77$. C: Immunoblotting of FAK and pFAK-Tyr397 in the presence or absence of serum and insulin. Equal amounts of protein were separated by SDS-PAGE and analyzed by immunoblotting with the indicated antibodies. D: Quantification of FAK and pFAK-Tyr397 signals from immunoblots. Blots from three independent experiments were quantified by densitometry. Signals were normalized to the signal from control (GFP-overexpressing) cells in full growth medium (+serum, +insulin). Data represent means \pm SEM. $n=10$ (GFP cells); $n=8$ (StARD3-GFP cells). ${ }^{*} P<0.05$ (Student's $t$-test), ${ }^{* * * *} P<$ 0.0001 (Mann-Whitney-Wilcoxon test) versus GFP control cell line; ${ }^{\dagger} P<0.05$ for -serum, - insulin versus full serum conditions (Student's $t$-test). Scale bar $=5 \mu \mathrm{m}$. minutes. Binding of the primary antibody was detected with a PowerVision+ Poly-HRP Histostaining Kit from ImmunoVision Technologies (Norwell, MA), following the manufacturer's recommendations. For both StARD3 and Src, cytoplasmic staining intensity was graded as negative, low, or high when at least $10 \%$ of the tumor cells showed protein expression (as is standard in the field). Nuclear staining was not considered relevant for this study. Example images of stained tissue were taken with a Leica DM LB microscope (Leica Microsystems) with an N Plan $20 \times$ objective, equipped with an Olympus DP50 color camera (Olympus Corporation).

For ErbB2 (HER-2) staining in the FinProg series, the sections were deparaffinized, followed by antigen retrieval (autoclave treatment at $121^{\circ} \mathrm{C}$ for 2 minutes in $10 \mathrm{mmol} / \mathrm{L}$ sodium citrate buffer, $\mathrm{pH}$ 6.0). The primary antibody, CB11 from Novocastra Laboratories (Newcastle upon Tyne, UK), was diluted 1:200 in Powervision blocking solution from Immunovision Inc. (Daly City, CA) and incubated overnight at $4^{\circ} \mathrm{C}$. An antimouse-peroxidase polymer from Powervision (30 minutes at room temperature) and diaminobenzidine chromogen were used for visualization. The sections were counterstained with hematoxylin and embedded. Positive and negative control samples (tumors with and without HER2 amplification in fluorescence in situ hybridization) were included in every staining batch. Evaluation of immunohistochemistry was done using a $20 \times$ objective. ErbB2 immunoreactivity was scored as negative, low, intermediate, or high, and only high (3+ like) staining was considered as ErB2 positive. ${ }^{22}$ In the FinHer series, ErbB2 protein expression was analyzed centrally using the HercepTest, as described elsewhere. ${ }^{24}$

Immunostaining for estrogen receptors was done on adjacent tissue array sections using the monoclonal antibody $6 \mathrm{~F} 11$ (Novocastra Laboratories; dilution, 1:500) and for progesterone receptors using the antibody 312 (Novocastra Laboratories; dilution, 1:500). Immunostaining was considered as positive when $>10 \%$ of cancer cells showed staining. The p53 was immunostained with the DO7 antibody (Novocastra Laboratories) at a dilution of 1:500 and Ki-67 using the MM-1 antibody (Novocastra Laboratories; dilution, 1:1000). Ki-67 and p53 staining were classified into three categories: i) negative (no positively staining cancer cell nuclei found), ii) borderline ( $\leq 20 \%$ nuclei positive), or iii) positive ( $>20 \%$ of cancer cell nuclei stained). Because there was no significant difference in outcome between patients who had either negative or borderline staining, these groups were combined in further analyses.

\section{Quantitative Real-Time RT-PCR}

Cells were homogenized in RLT buffer from QIAGEN Sciences (Gaithersburg, MD), and total RNA was isolated with the RNeasy Mini Kit (74104) from QIAGEN Sciences, according to the manufacturer's instructions. The total RNA $(1 \mu \mathrm{g})$ was reverse transcribed, as previously described. ${ }^{25}$ 3-Hydroxy-3methylglutaryl-coenzyme A reductase (HMGR) cDNA was quantified using LightCycler 480 Probe 85 (04689097001) from Roche Diagnostics (Basel, Germany). TATAA binding 


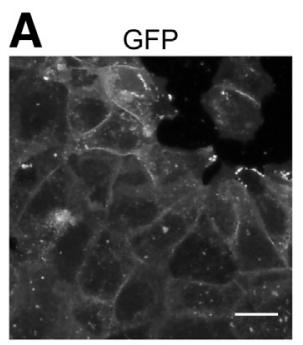

Filipin

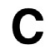

C

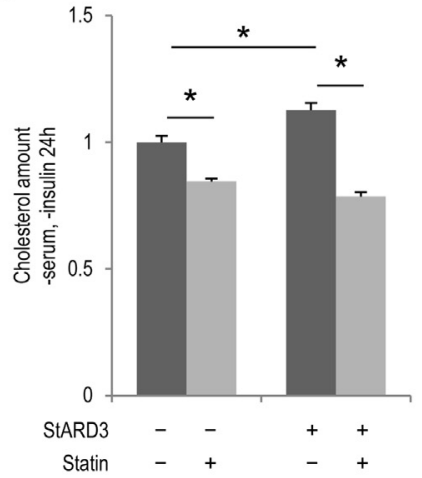

StARD3-GFP

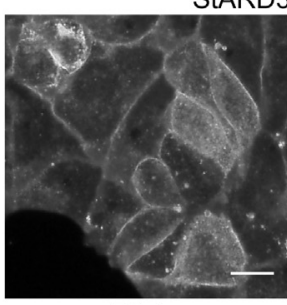

Filipin

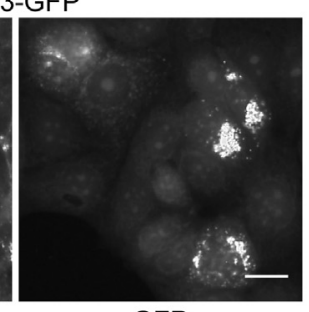

GFP

D

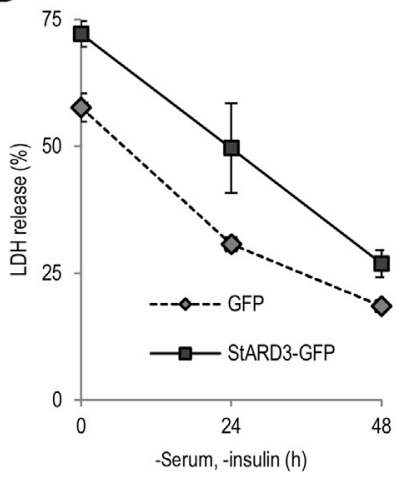

B

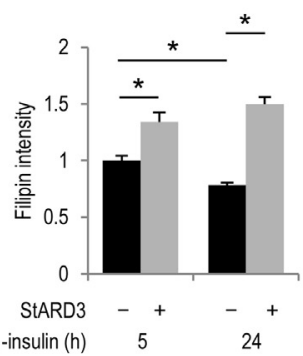

E

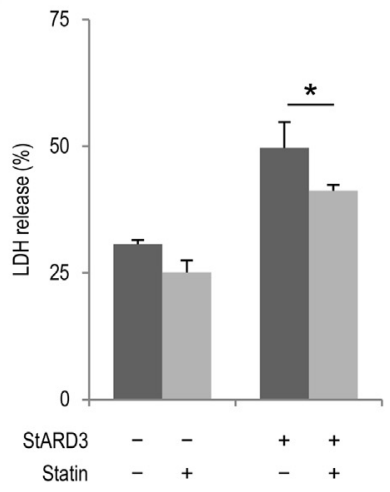

Figure 5 Effects of StAR-related lipid transfer protein 3 (StARD3) overexpression on cholesterol balance in serum- and insulin-deprived conditions. A: Filipin staining of cells deprived of serum and insulin for 24 hours. Cells were fixed with paraformaldehyde, stained with filipin, and imaged by wide-field fluorescence microscopy. Please note higher filipin intensity in cells with prominent StARD3-green fluorescent protein (GFP) expression. B: Fluorescence intensity quantification from cells deprived of serum and insulin. GFP and StARD3-GFP cells were deprived of serum and insulin for 5 or 24 hours, fixed, stained with filipin, and imaged as in A, using the same settings for all conditions. The total filipin fluorescence intensity per cell was measured for 30 to 40 cells per condition and normalized to control cells deprived of serum and insulin for 5 hours. C: Total cholesterol content in cells deprived of serum and insulin. GFP and StARD3-GFP cells were deprived of serum and insulin in the presence or absence of lovastatin for 24 hours before lipid analysis. D: Sensitivity of cells to streptolysin 0 (SLO) permeabilization on serum and insulin deprivation for 0,24 , or 48 hours. Resistance of the plasma membrane to SLO was measured as the fraction of total cellular lactate dehydrogenase (LDH) released to the medium after 30 minutes of treatment at $37^{\circ} \mathrm{C}$. E: Sensitivity of cells to SLO permeabilization on serum and insulin deprivation for 24 hours in the presence or absence of lovastatin. D: Values in the presence of lovastatin were normalized to the values in the absence of lovastatin at 24 hours of serum and insulin deprivation. Data represent means \pm SEM (B); data represent means \pm SEM of cholesterol $(\mathrm{ng})$ per protein $(\mu \mathrm{g})(\mathbf{C})$; data represent means $\pm S D$ of triplicate measurements (D). $n=6$ to 10 from three independent experiments, cholesterol amounts normalized to GFP control within

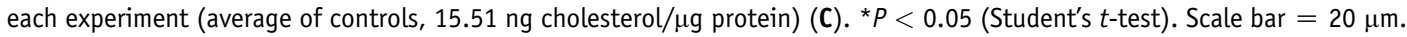

box protein was used as an internal control for normalization. Human adrenal tissue was used as a positive control for the cell and breast cancer tissue samples, and RNA and cDNA were generated as for cells. Each sample was run in triplicate. Normalized gene expression values were obtained using LightCycler 480 software version 1.5.1 from Roche Applied Science (Mannheim, Germany), using the basic relative quantification method. The analysis of the patient samples was done identically, but RNA was extracted by using the Versant kPCR Molecular System (Siemens AG, Erlangen, Germany) and normalized gene expression values were obtained using the LightCycler advanced quantification method, as described in detail previously. ${ }^{26}$ Adrenal tissue RNA served as a calibrator sample and was used to construct the standard curves for the target and the reference genes.

\section{Statistical Analysis}

All data assumed to have a normal distribution were represented as bar charts, with the height of the bar representing the mean and the error bar representing the SEM. Tests of the statistical significance of normally distributed values were performed using a nonpaired two-tailed Student's $t$-test. Continuous distributions of nonnormally distributed values were summarized in box-and-whiskers plots, with the bottom and top of the box representing the first and third quartiles, respectively, and the band inside the box representing the median. The ends of the whiskers represent the lowest datum still within 1.5 times the interquartile range of the lower quartile, and highest datum still within 1.5 times the interquartile range of the upper quartile. Data points outside of these ranges were not included in the box plots. For nonnormally distributed values, statistical significance was calculated using the Mann-Whitney-Wilcoxon rank sum test. Statistical significance for frequency tables was calculated using the $\chi^{2}$ test.

An analysis of breast cancer-specific survival was available for the patients included in the FinProg series, and was calculated from the date of breast cancer diagnosis to death from breast cancer, censoring patients who were alive on the date of the last follow-up and those who died from a competing cause on the date of death. In the FinHer series, 
A StARD3 Ab B
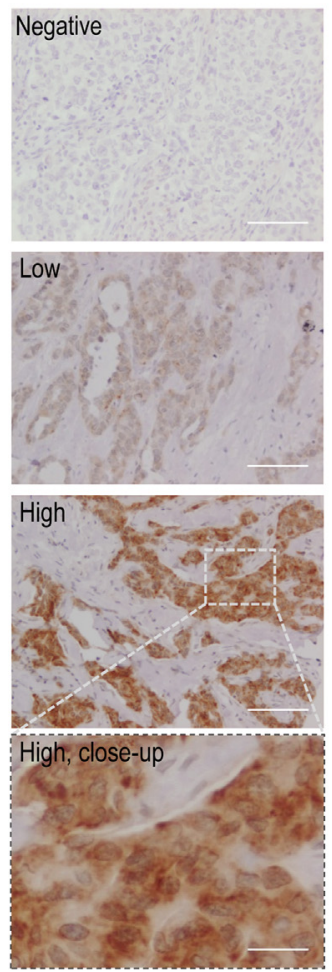

B

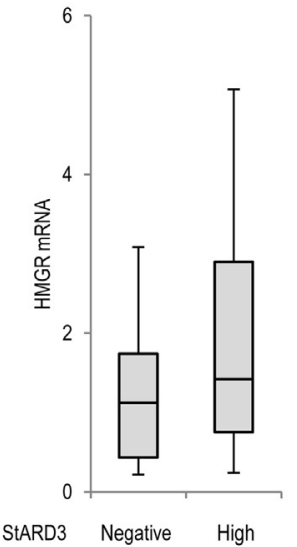

C

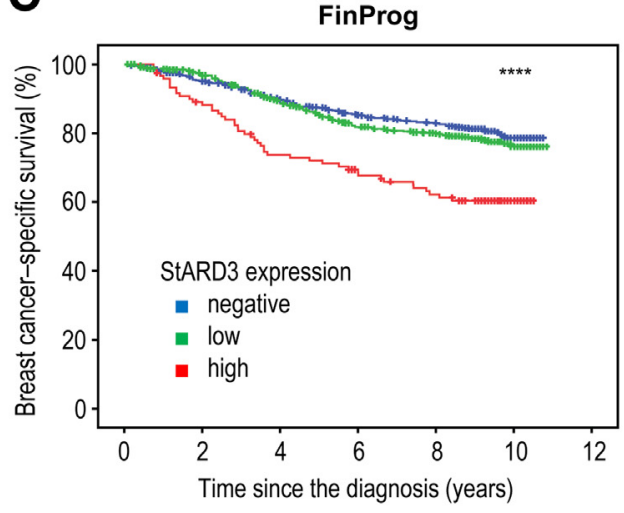

FinHer

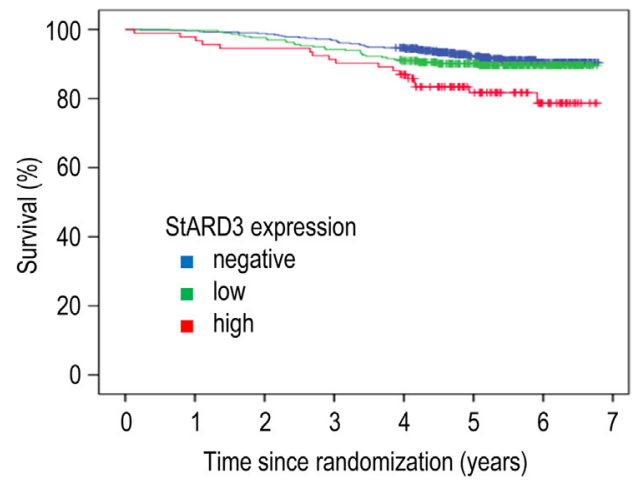

Figure 6 High StAR-related lipid transfer protein 3 (StARD3) protein levels in breast cancer correlate with increased 3-hydroxy-3-methylglutaryl-coenzyme A reductase (HMGR) mRNA levels and decreased patient survival. A: StARD3 immunohistochemistry of primary breast cancers. Top three panels: Exemplary micrographs of negative, low, or high StARD3 levels. Bottom panel: a close-up of the indicated area from high expression level. B: HMGR mRNA levels in tumors with negative or high StARD3 expression. Total RNA extracted from cells was reverse transcribed, and HMGR cDNA was quantified by real-time RT-PCR. Bars represent HMGR transcript levels and are normalized using HMGR levels in control tissue (adrenal cortex). Box plots: StARD3-negative samples ( $n=31$, median $=1.12$ ) and high StARD3 samples $(n=38$, median $=1.42) . P=0.048$ (Mann-Whitney-Wilcoxon test). Outliers, as defined in Materials and Methods, are not shown on the plot. C: Kaplan-Meier analysis for breast cancer-specific survival of patients in FinProg cohort with negative, low, or high StARD3 protein levels and Kaplan-Meier analysis for end point overall survival of patients with StARD3 protein levels available in FinHer cohort. Negative, low, or high StARD3 expression. $P=0.007$ (log-rank test) when all three categories are included. $* * * * P<0.0001$ (log-rank test) when all three categories are included. Scale bar $=100 \mu \mathrm{m}(\mathbf{A}) ; 25 \mu \mathrm{m}$ (bottom panel, A).

overall survival was calculated from the date of randomization to the date of death, censoring patients who were alive on the date of the last follow-up visit. Survival between groups was calculated using the log-rank test. The $P$ values are two sided.

\section{Results}

\section{Stable 0verexpression of StARD3 in MCF-7 Cells Alters} Cell Morphological Features

To address the role of StARD3 protein in breast cancer cells and tissues, we generated polyclonal rabbit antibodies against the START domain of human StARD3. The affinity-purified antibodies were specific for StARD3 because the immunoreactivity was significantly reduced on transient StARD3 silencing from KPL-4 and BT-474 cells, both of which express StARD3 and ErbB2 at relatively high levels ${ }^{27}$ (Supplemental Figure S1, A-D). Furthermore, we found that knockdown of StARD3 in BT-474 cells decreased cell survival (Supplemental Figure S1,
E and F). As expected, ErbB2 silencing also decreased cell survival (Supplemental Figure S1, E and F). These observations suggest that both ErbB2 and StARD3 promote the survival of breast cancer cells, and are in agreement with earlier findings. ${ }^{4}$

To gain insights into how StARD3 may promote cell survival independently of $H E R 2$ amplification, we generated MCF-7 (HER2-negative) breast cancer cells that stably overexpress StARD3-GFP or soluble GFP as a control. Overexpression of StARD3-GFP and GFP proteins was verified by Western blot analysis from cell lysates (Figure 1A). Immunoblotting also revealed that the ErbB2 protein levels were not increased on StARD3-GFP overexpression (Figure 1A). Thus, in this cell line, the effects of StARD3 overexpression can be addressed in the absence of HER2 overexpression. Confocal microscopy showed that control GFP expression alone was cytosolic, as expected (Figure 1B), and that the overexpressed StARD3-GFP protein exhibited a punctate distribution, colocalizing, in part, with the late endosomal marker, Lamp-1 (Figure 1B). 
Table 1 Summary of StARD3 Immunoreactivity in the Analyzed Patient Cohorts, with Number of Tumors and Distribution of StARD3 Intensity

\begin{tabular}{llll}
\hline \multirow{2}{*}{ Study } & \multicolumn{3}{l}{ StARD3 overexpression (\%) } \\
\cline { 2 - 4 } & Negative & Low & High \\
\hline FinProg $(n=1325)$ & $598(45.1)$ & $607(45.8)$ & $120(9.1)$ \\
FinHer $(n=895)$ & $507(56.6)$ & $296(33.1)$ & $92(10.3)$ \\
\hline
\end{tabular}

StARD3, StAR-related lipid transfer protein 3.

In addition, some of the StARD3-GFP localized to the plasma membrane, as suggested by the prominent surface fluorescence observed in a fraction of cells (Figure 1, $\mathrm{C}$ and $\mathrm{D})$. Total internal reflection fluorescence microscopy revealed a punctate pattern of StARD3-GFP fluorescence in the plasma membrane plane, reflective of plasma membrane domains and/or small vesicles immediately underneath. These results are in accordance with our previous observations on StARD3 subcellular localization. ${ }^{14}$

Remarkably, the overall morphological features of the StARD3-GFP-overexpressing cells were strikingly different from those of control GFP cells. The StARD3-GFP cells grew in clusters of rounded-up cells that did not adhere well to the substratum and lacked growth contact inhibition, as demonstrated by phase-contrast and GFP-fluorescence images of living cells (Figure 1C). Such growth pattern is often characteristic of malignant cells.

\section{StARD3 Overexpression Results in Altered Cellular Cholesterol Balance}

To study whether the overexpression of StARD3-GFP affects cellular cholesterol homeostasis, we stained StARD3GFP- and GFP-expressing cells with filipin, a fluorescent sterol-binding compound that stains cholesterol (and other sterols with a 3 -carbon $\mathrm{OH}$-group) in cells. ${ }^{28} \mathrm{We}$ found that StARD3-GFP cell clusters showed increased filipin intensity, especially in the plasma membrane, as compared to control cells (Figure 2A). This suggests the possibility that the cholesterol content of StARD3-overexpressing cells may be elevated. However, on the basis of biochemical cholesterol determination, the amount of total cellular-free cholesterol in StARD3-GFP cells was not increased, but rather slightly decreased, as compared to control cells (Figure 2B). Therefore, the increased filipin staining intensity in StARD3-overexpressing cells more likely reflects an altered cellular cholesterol distribution. This idea was corroborated by the finding that the level of HMGR mRNA was increased in StARD3-overexpressing cells (Figure 2C). HMGR, the rate-limiting enzyme of cholesterol biosynthesis, is located in the ER and known to be up-regulated on ER cholesterol depletion. ${ }^{29}$ To further study if the ER of StARD3-overexpressing cells is cholesterol poor, we analyzed the levels and processing of the key transcriptional regulator of cholesterol metabolism, sterol regulatory element-binding protein 2 . Under ER cholesterol depletion, sterol regulatory element-binding protein 2 is increased and its processing to the mature, transcriptionally active form is enhanced. We found this to be the case in StARD3-GFP-overexpressing cells compared to control cells (Figure 2, D and E). Thus, in StARD3-overexpressing cells, the ER appeared to be cholesterol poor and cholesterol synthesis elevated.

To investigate whether the plasma membrane cholesterol content was altered on StARD3 overexpression, we measured the resistance of the cells to SLO. This sterolbinding bacterial toxin forms pores in cholesterol-rich plasma membrane regions. ${ }^{30}$ The fraction of cytosolic LDH activity released from cells is used as a measure of membrane permeabilization and, indirectly, as a measure for membrane cholesterol content. As expected, in both GFP- and StARD3-GFP-overexpressing cells, SLO induced the release of LDH from cells (Figure 2F). More important, LDH release from StARD3-GFP-overexpressing cells was higher than that from GFP-expressing cells (1.16-fold; average normalized to GFP control in both SLO concentrations, $\mathrm{SD}=0.076$ ). This strongly suggests that the plasma membrane cholesterol content of StARD3overexpressing cells is higher than that of control cells. Together, these results provide evidence that StARD3 overexpression leads to alterations in cellular cholesterol distribution and homeostatic control, with increased plasma membrane cholesterol but decreased ER cholesterol.

\section{The Effects of StARD3 Overexpression on Cell Morphological Features Are Serum and Insulin Dependent}

While studying the morphological effects of StARD3-GFP overexpression, we noticed a striking phenotypic change in StARD3-GFP cells when serum and insulin were deprived from the culture medium. Already within 5 hours after the removal of these growth stimuli, more than half of the StARD3-overexpressing cells became clearly more adherent (Figure 3A). In 24 hours, most of the StARD3-GFP cells had adhered tightly to the substratum and their morphological appearance was more closely reminiscent of that of control GFP cells (Figure 3A). In contrast, serum and insulin withdrawal had no apparent effects on the morphological features of the GFP cells within this time frame (Figure 3A). This change in the morphological features of StARD3-overexpressing cells did not result from reduced StARD3-GFP protein levels on serum and insulin deprivation, as evidenced by Western blot analysis (Figure 3B). In fact, the StARD3-GFP protein levels increased under these conditions (Figure 3, B and C), probably because of increased protein stability, considering that the StARD3GFP expression was under a viral promoter. Because the proto-oncogene Src is a key regulator of cell adhesion and implicated in breast cancer development, ${ }^{31}$ we analyzed its levels. This showed that Src levels were moderately 
Table 2 Association of StARD3 Immunoreactivity with Histopathological Parameters in FinProg Material

\begin{tabular}{|c|c|c|c|}
\hline \multirow[b]{2}{*}{ Parameter } & \multicolumn{3}{|c|}{ StARD3 overexpression (\%) } \\
\hline & Negative & Low & High \\
\hline \multicolumn{4}{|l|}{ ErbB2 immunostaining } \\
\hline Negative & $503(93.5)$ & $445(82.7)$ & $27(23.1)$ \\
\hline Positive & $35(6.5)$ & $93(17.3)$ & $90(76.9)$ \\
\hline \multicolumn{4}{|l|}{ ERBB2-CISH } \\
\hline Negative & $491(92.8)$ & $437(82.1)$ & $20(17.4)$ \\
\hline Positive & $38(7.2)$ & $95(17.9)$ & $95(82.6)$ \\
\hline \multicolumn{4}{|l|}{ Molecular subtype } \\
\hline Luminal A & $377(75.6)$ & $344(69.4)$ & $14(13.7)$ \\
\hline Luminal B & $23(4.6)$ & $50(10.1)$ & $34(33.3)$ \\
\hline $\mathrm{HER}^{+} / \mathrm{HR}^{-}$ & $12(2.4)$ & $40(8.1)$ & $48(47.1)$ \\
\hline Basal-like & $60(12.0)$ & $35(7.1)$ & $1(1.0)$ \\
\hline Nonexpressor & $27(5.4)$ & $27(5.4)$ & $5(4.9)$ \\
\hline \multicolumn{4}{|l|}{ pSrc } \\
\hline Negative & $504(90.8)$ & $422(76.6)$ & $85(73.9)$ \\
\hline Positive & $51(9.2)$ & $129(23.4)$ & $30(26.1)$ \\
\hline \multicolumn{4}{|l|}{ Estrogen receptor } \\
\hline Negative $(<20 \%)$ & $155(29.1)$ & $159(29.7)$ & $66(60.0)$ \\
\hline Positive $(>20 \%)$ & $377(70.9)$ & $376(70.3)$ & $44(40.0)$ \\
\hline \multicolumn{4}{|l|}{ p53 } \\
\hline Negative $(<20 \%)$ & $420(84.5)$ & $383(80.3)$ & $63(61.8)$ \\
\hline Positive $(>20 \%)$ & $77(15.5)$ & $94(19.7)$ & $39(38.2)$ \\
\hline \multicolumn{4}{|l|}{$\mathrm{PgR}$} \\
\hline Negative $(<20 \%)$ & $226(41.9)$ & $234(44.3)$ & $84(76.4)$ \\
\hline Positive $(>20 \%)$ & $313(58.1)$ & $294(55.7)$ & $26(23.6)$ \\
\hline \multicolumn{4}{|l|}{ Ki-67 } \\
\hline Negative $(<20 \%)$ & $341(68.2)$ & $309(60.8)$ & $38(38.4)$ \\
\hline Positive $(>20 \%)$ & $159(31.8)$ & $199(39.2)$ & $61(61.6)$ \\
\hline \multicolumn{4}{|c|}{ Tumor size distribution* } \\
\hline Min & $1(0.6)$ & & $3(2.5)$ \\
\hline Max & $140(87.0)$ & & $90(76.3)$ \\
\hline Median & $20(12.4)$ & & $25(21.2)$ \\
\hline \multicolumn{4}{|l|}{ Age distribution* } \\
\hline Min & $23(12.9)$ & & $27(15.9)$ \\
\hline Max & $96(53.9)$ & & $90(52.9)$ \\
\hline Median & $59(33.1)$ & & $53(31.2)$ \\
\hline \multicolumn{4}{|c|}{ Nodal metastases $(P=0.014)$} \\
\hline No & $384(65.4)$ & $368(62.5)$ & $61(51.3)$ \\
\hline Yes & $203(34.6)$ & $221(37.5)$ & $58(48.7)$ \\
\hline \multicolumn{4}{|l|}{ Histological features } \\
\hline Ductal & $433(72.4)$ & $470(77.4)$ & $102(85.0)$ \\
\hline Lobular & $121(20.2)$ & $69(11.4)$ & $8(6.7)$ \\
\hline Other & $44(7.4)$ & $68(11.2)$ & $10(8.3)$ \\
\hline \multicolumn{4}{|l|}{ Grade } \\
\hline I & $118(27.1)$ & $108(23.5)$ & $6(6.1)$ \\
\hline II & $212(48.7)$ & $211(46.0)$ & $53(53.5)$ \\
\hline III & $105(24.1)$ & $140(30.5)$ & $40(40.4)$ \\
\hline
\end{tabular}

$P<0.0001$, in all parameters except nodal metastases; Mann-Whitney test for statistical significance was used for tumor size and age distributions.

*StARD3 columns negative and low combined.

CISH, chromogenic in situ hybridization; HR, hormone receptor; Max, maximum; Min, minimum; PgR, progesterone receptor; StARD3, StARrelated lipid transfer protein 3.

elevated in StARD3-overexpressing cells in complete medium conditions relative to control cells and stayed high in both control and StARD3-overexpressing cells when serum and insulin were removed (Figure 3D).
StARD3-0verexpressing Cells Exhibit Altered FAs and FA Signaling in Serum- and Insulin-Deprived Conditions

To further investigate the molecular basis for the differences in the adherence between GFP and StARD3-GFP cells, we imaged cellular FAs. The StARD3-overexpressing cells were largely rounded up and depleted of FAs in complete medium (Figures 1C and 3A). However, these cells developed prominent, irregularly shaped adhesions under serum and insulin deprivation, as revealed by anti-FAK antibody staining (Figure 4A). Quantification of the area occupied by antibody staining showed that the mean area of FAs was roughly doubled and the variability in FA size was increased on StARD3 overexpression (Figure 4B). Considering that FAK is a key substrate of Src kinase, we analyzed the levels and phosphorylation status of FAK by immunoblotting. This showed that in control cells, there was no substantial difference in FAK protein levels or phosphorylation at Tyr397 on serum and insulin withdrawal (Figure 4, C and D). Although there was a tendency for increased FAK signal, the level and degree of Tyr397 phosphorylation of FAK were not significantly different between control and StARD3-overexpressing cells in complete medium. However, on serum and insulin removal, both the total levels of FAK and its phosphorylation at Tyr397 were significantly increased in StARD3-overexpressing cells compared to control cells (Figure 4, C and D). These results suggest that altered signaling at FAs contributes to the improved adherence of StARD3-overexpressing cells on removal of the growth stimuli.

\section{StARD3-0verexpressing Cells Maintain Increased Cellular and Plasma Membrane Cholesterol Content in Serum- and Insulin-Deprived Conditions}

Considering that StARD3 overexpression altered the plasma membrane cholesterol content and that this is known to affect cell adhesion, ${ }^{32}$ we investigated how serum and insulin deprivation affected the plasma membrane cholesterol content in control and StARD3-overexpressing cells. The cells were incubated for 5 or 24 hours in the absence of these growth stimuli and stained with filipin, and the fluorescence intensity of individual adherent cells was analyzed. This was considerably easier to perform now that the StARD3 cells were no longer in rounded-up clusters and the cell boundaries were better visualized.

We found that cells overexpressing StARD3-GFP at high levels showed a robust increase in filipin intensity compared to control GFP cells or cells that overexpressed StARD3GFP at more moderate levels (Figure 5, A and B). This effect was evident both at 5 and 24 hours of serum and insulin deprivation (Figure 5B). Moreover, although the filipin intensity of control GFP cells decreased from 5 to 24 hours, that of StARD3-GFP cells did not (Figure 5B). These results suggest that the plasma membrane cholesterol content of control cells decreased when they were serum deprived, in 
Table 3 Association of StARD3 Immunoreactivity with Histopathological Parameters in FinHer Material

\begin{tabular}{lcrc}
\hline & \multicolumn{3}{l}{ StARD3 overexpression (\%) } \\
\cline { 2 - 4 } Parameter & Negative & Low & High \\
\hline HercepTest & & & \\
$\quad 0$ or 1+ & $473(96.7)$ & $168(58.1)$ & $3(3.3)$ \\
$2+$ & $12(2.5)$ & $73(25.3)$ & $26(28.6)$ \\
$3+$ & $4(0.8)$ & $48(16.6)$ & $62(68.1)$ \\
ERBB2-CISH & & & \\
$\quad$ Negative & $483(95.3)$ & $199(67.2)$ & $9(9.8)$ \\
$\quad$ Positive & $24(4.7)$ & $97(32.8)$ & $83(90.2)$ \\
pSrc & & & \\
$\quad$ Negative & $361(64.3)$ & $105(47.3)$ & $19(23.2)$ \\
$\quad$ Positive & $200(35.7)$ & $117(52.7)$ & $63(76.8)$ \\
Estrogen receptor & & & \\
$\quad$ Negative $(<20 \%)$ & $110(21.7)$ & $85(28.7)$ & $58(63.0)$ \\
$\quad$ Positive $(>20 \%)$ & $397(78.3)$ & $211(71.3)$ & $34(37.0)$ \\
p53 & & & \\
$\quad$ Negative $(<20 \%)$ & $379(79.1)$ & $209(72.6)$ & $46(51.1)$ \\
$\quad$ Positive $(>20 \%)$ & $100(20.9)$ & $79(27.4)$ & $44(48.9)$ \\
\hline
\end{tabular}

$P<0.0001$ in all cases.

CISH, chromogenic in situ hybridization; StARD3, StAR-related lipid transfer protein 3 .

accordance with the notion that serum lipoproteins are a major source of cellular membrane cholesterol. In contrast, the StARD3-GFP cells seemed to be capable of maintaining elevated plasma membrane cholesterol levels in the absence of serum and insulin.

Biochemical cholesterol determinations showed that serumand insulin-starved StARD3-GFP cells maintained an elevated cholesterol content compared to control GFP cells (Figure 5C). To test if this was due to increased cholesterol synthesis, we treated the cells with lovastatin, an inhibitor of HMGR, during the 24-hour serum and insulin deprivation. Statin treatment abolished the capability of StARD3-GFP cells to elevate their cholesterol content (Figure 5C), implying that increased cholesterol synthesis accounted for the elevated cholesterol levels in StARD3-GFP-expressing cells. Moreover, the plasma membrane cholesterol amounts of StARD3-GFP cells were higher than those of control cells after 24 to 48 hours of serum and insulin deprivation, as assessed by sensitivity to SLO permeabilization (Figure 5D). This was also repressed by statin, as expected (Figure 5E).

High StARD3 Protein Levels in Human Breast Tumor Samples Associate with ERBB2 Amplification, Cholesterol Imbalance, and Src Activation

To investigate StARD3 protein expression in Finnish breast cancer patients, we performed immunohistochemical staining of 1325 primary breast cancer samples deriving from the FinProg cohort $^{22}$ using our anti-StARD3 affinity-purified antibody. The StARD3 staining intensity was graded as negative, low, or high (Figure 6A). Stromal cells were not stained, and normal epithelial cells showed negative or low staining. In cells with high staining intensity, the staining exhibited a perinuclear, dot-like staining pattern, with nuclei devoid of staining (Figure 6A). These observations are in concordance with previous results. ${ }^{8,33}$ We found that 120 $(9.1 \%)$ of the samples exhibited high StARD3 staining, 607 (45.8\%) showed low StARD3 staining, and 598 (45.1\%) were negative (Table 1). High StARD3 immunoreactivity was associated with ErbB2 immunoreactivity and HER2 amplification, and several factors related to a poor disease outcome, such as high proliferation rate, bigger tumor size, and nodal metastases at the time of diagnosis, as well as estrogen and progesterone receptor negativity and $\mathrm{p} 53$ protein expression (Table 2). Considering that our in vitro data suggested high StARD3 expression to be associated with increased Src-FAK signaling, we also analyzed the tumor samples by anti-Src Tyr416 (pSrc) staining, which provides a measure of Src activation. We found that in the FinProg material, $1011(82.8 \%)$ of the samples scored as negative and $210(17.2 \%)$ positive for pSrc staining, and that positive Src phosphorylation associated with high StARD3 protein expression (Table 2).

To address StARD3 and pSrc immunoreactivity in an independent patient cohort, we analyzed 895 primary breast cancer tissue specimen from the FinHer study. ${ }^{23}$ We found that $92(10.3 \%)$ of these samples exhibited high StARD3 staining, 296 (33.1\%) showed low StARD3 staining, and $507(56.6 \%)$ were negative (Table 1). For pSrc, $485(56.1 \%)$ of the FinHer samples were negative, and $380(43.9 \%)$ were positive. In this cohort, high StARD3 immunostaining was strongly associated with HER2 gene amplification, ErbB2 immunoreactivity, and pSrc positivity, as well as hormone receptor negativity and p53 expression (Table 3). From a subset of the FinHer material, RNA was available and we could analyze HMGR mRNA levels. The subset consisted of 38 primary breast tumors scored as highly StARD3 positive and 31 tumors that were scored StARD3 negative by immunostaining. The analysis showed that the highest HMGR transcript levels were found in the highly StARD3positive tumors (Figure 6B). Together, these findings suggest that elevated StARD3 protein expression was accompanied by increased cholesterol synthesis and Src activation in human breast cancers. Finally, our analyses showed that high StARD3 protein level was strongly associated with decreased cumulative patient survival in both FinProg $(P<0.0001)$ and FinHer $(P=0.007)$ cohorts (Figure 6C).

\section{Discussion}

This study was conducted to address how elevated StARD3 protein expression affects breast cancer cells and to delineate parameters characteristic of primary human breast cancers that exhibit high StARD3 protein content. This is of interest because StARD3 has been shown to be coamplified and co-overexpressed as part of the HER2/ERBB2 17q12 amplicon in a subset of breast cancers. ${ }^{4,17,33-35}$ Moreover, anti-ErbB2 antibody therapy is widely used in the clinical 
setting, yet $50 \%$ or even more of ErbB2-positive patients show no response, or become resistant to the treatment. ${ }^{36}$ It has been proposed that some of the coamplified gene products may contribute to cancer progression. ${ }^{3,4,7}$ StARD3 is an attractive candidate for such a modulator, because as earlier data and our current results in two Finnish patient cohorts indicate, the gene is practically invariably coamplified and the protein co-overexpressed with ErbB2 in HER2-positive breast cancers.

StARD3 is a cholesterol-binding protein with a 1:1 stoichiometry ${ }^{10}$ and has been shown to affect intracellular membrane and cholesterol transport. ${ }^{11,14,15}$ Cancers, including breast cancer, have been associated with alterations in cholesterol metabolism, ${ }^{37}$ with both cholesterol and its metabolites being capable of deregulating breast cancer cells. ${ }^{38}$ Cancer cells require a continued supply of cholesterol for membrane biogenesis, to support cell growth and division. In addition, cholesterol plays an important role in organizing cholesterol-sphingolipid-rich membrane domains, lipid rafts, that are involved (e.g., in cell signaling and adhesion). ${ }^{39}$ Alterations in the plasma membrane cholesterol content affect cell signaling, adhesion, and motility. ${ }^{40-42}$ These processes are fundamental to the survival and growth of cancer cells as well as their ability to metastasize.

To dissect the potential ErbB2-independent role of elevated StARD3 protein content in breast cancer cells, we generated MCF-7 cells overexpressing StARD3. On the basis of our results, overexpressing StARD3 caused several alterations in cellular cholesterol homeostasis that are potentially cancer promoting. The StARD3-overexpressing cells had increased levels of the mRNA encoding the ratelimiting cholesterol biosynthesis enzyme HMGR. Increased cholesterol biosynthesis allows cells to survive and to keep dividing, especially in low nutrient conditions, where membrane biogenesis is normally limited. In addition, the StARD3-overexpressing cells exhibited increased plasma membrane cholesterol levels. Moreover, the cholesterol content of StARD3-overexpressing cells was refractory to the withdrawal of the exogenous cholesterol source (ie, serum lipoproteins). Compared to control cells, the StARD3-overexpressing cells managed to maintain elevated total and plasma membrane cholesterol levels in the absence of serum due to the up-regulated cholesterol biosynthesis. The sterol-binding compounds used, filipin and SLO, also bind cholesterol precursor sterols that are up-regulated during cholesterol biogenesis. Thus, these may contribute to the increased sterol content.

A high plasma membrane cholesterol level supports raftdependent signaling via lipid-anchored proteins, such as Src family kinases. This, in turn, alters the adhesive properties of cells. ${ }^{43}$ Indeed, we observed that StARD3 overexpression affected cell adherence: the cells were globular and loosely attached to the substratum in the presence of serum and insulin, but became adhesive on their withdrawal. Consistently, the cells displayed exaggerated FAs and increased phosphorylation of FAK, a major Src substrate. It could be envisioned how such alterations in cell morphological features might, on one hand, favor shedding of cancer cells to the bloodstream and, on the other hand, allow cells to adhere tightly to the underlying tissue and endure limited nutrient conditions within a solid tumor.

There are several mechanisms by which StARD3 could increase plasma membrane cholesterol. First, the newly synthesized StARD3 protein moves to the LEs via the plasma membrane. ${ }^{16}$ Increased StARD3 production is likely to increase the amount of StARD3 in the plasma membrane, where it may enhance cholesterol deposition similarly as we have shown for LEs. ${ }^{14}$ Second, increased cholesterol in StARD3-containing LEs may become transported to the plasma membrane. The LEs communicate actively with the plasma membrane, and we have recently demonstrated that LE-to-plasma membrane cholesterol transport is an important regulator of cell migration. ${ }^{42}$ Finally, newly synthesized cholesterol is efficiently delivered from the ER to the plasma membrane. ${ }^{44}$ Thus, increased cholesterol biosynthetic activity observed in StARD3-overexpressing cells probably also facilitates plasma membrane cholesterol enrichment.

In two large nationwide cohorts, FinProg and FinHer, with a total of $>2000$ breast cancer patients, we observed that $H E R 2$ gene amplification and ErB2 immunoreactivity were tightly associated with high StARD3 protein expression. This subgroup of highly StARD3-positive tumors was composed of approximately 1 of 10 patients in both cohorts. In fact, we could not identify patients who would have high StARD3 immunoreactivity without HER2 amplification. Hence, we cannot make statements about the specific role of high StARD3 expression alone in the clinical setting. Nevertheless, we found that high StARD3 protein levels correlated with elevated HMGR mRNA levels in the FinHer cohort, in accordance with the in vitro cell data. Moreover, high StARD3 protein levels associated with high levels of Src phosphorylation on the activating Tyr416 in both FinProg and FinHer cohorts. This, together with the cell data that show increased Src, elevated phosphorylation of the downstream kinase FAK, and enlarged FAs on StARD3 overexpression in the absence of HER 2 amplification; these findings fit with the idea that high StARD3 protein levels promote Src signaling.

More important, Src activation appears as a key contributor to trastuzumab resistance in HER2-positive cancers, ${ }^{45,46}$ because patients with active Src were found to be significantly less responsive to trastuzumab than those without Src activation. Although ErbB2 itself is linked to Src activation, other candidates in the HER2 amplicon have not, to our knowledge, been directly implicated in this process. Our study provides evidence that elevated StARD3 expression is linked to increased Src activity and points to the possibility that StARD3 may contribute to the aggressive behavior of trastuzumab-resistant tumors. Therefore, it would be warranted to investigate if repression of StARD3 activity (e.g., by using small-molecule inhibitors) might be 
useful in HER2-positive cancers and could be used to combat trastuzumab resistance.

In conclusion, we show that overexpression of STARD3, a gene coamplified with $H E R 2$, results in increased levels of plasma membrane cholesterol and Src, altered adhesiveness, and FAK signaling in HER2-negative breast cancer cells in vitro. These findings suggest that elevated StARD3 expression has a potential to contribute to breast cancer cell aggressiveness, by increasing membrane cholesterol and thereby enhancing oncogenic signaling. The clinical correlations were consistent because approximately $10 \%$ of the analyzed 2220 human breast cancers had high StARD3 protein levels, which associated with high ErbB2 levels, increased Src activity, and poor patient survival. Inhibition of StARD3 function might, therefore, be beneficial in HER2-positive cancers.

\section{Acknowledgments}

We thank Anna Uro and Pipsa Kaipainen for expert technical assistance.

\section{Supplemental Data}

Supplemental material for this article can be found at http://dx.doi.org/10.1016/j.ajpath.2014.12.018.

\section{References}

1. Kauraniemi P, Kallioniemi A: Activation of multiple cancer-associated genes at the ERBB2 amplicon in breast cancer. Endocr Relat Cancer 2006, 13:39-49

2. Staaf J, Jonsson G, Ringner M, Vallon-Christersson J, Grabau D, Arason A, Gunnarsson H, Agnarsson BA, Malmstrom PO, Johannsson OT, Loman N, Barkardottir RB, Borg A: High-resolution genomic and expression analyses of copy number alterations in HER2amplified breast cancer. Breast Cancer Res 2010, 12:R25

3. Kao J, Pollack JR: RNA interference-based functional dissection of the $17 q 12$ amplicon in breast cancer reveals contribution of coamplified genes. Genes Chromosomes Cancer 2006, 45:761-769

4. Sahlberg KK, Hongisto V, Edgren $H$, Mäkelä R, Hellström K, Due EU, Moen Vollan HK, Sahlberg N, Wolf M, Børresen-Dale A-L, Perälä M, Kallioniemi O: The HER2 amplicon includes several genes required for the growth and survival of HER2 positive breast cancer cells. Mol Oncol 2013, 7:392-401

5. Hynes NE, Lane HA: ERBB receptors and cancer: the complexity of targeted inhibitors. Nat Rev Cancer 2005, 5:341-354

6. Yarden Y: Biology of HER2 and its importance in breast cancer. Oncology 2001, 61(Suppl 2):1-13

7. Jacot W, Fiche M, Zaman K, Wolfer A, Lamy P-J: The HER2 amplicon in breast cancer: topoisomerase IIA and beyond. Biochim Biophys Acta 2013, 1836:146-157

8. Moog-Lutz C, Tomasetto C, Régnier $\mathrm{CH}$, Wendling C, Lutz Y, Muller D, Chenard MP, Basset P, Rio MC: MLN64 exhibits homology with the steroidogenic acute regulatory protein (STAR) and is overexpressed in human breast carcinomas. Int J Cancer 1997, 71:183-191

9. Alpy F, Tomasetto C: MLN64 and MENTHO, two mediators of endosomal cholesterol transport. Biochem Soc Trans 2006, 34:343-345

10. Tsujishita Y, Hurley JH: Structure and lipid transport mechanism of a StAR-related domain. Nat Struct Biol 2000, 7:408-414
11. van der Kant R, Zondervan I, Janssen L, Neefjes J: Cholesterolbinding molecules MLN64 and ORP1L mark distinct late endosomes with transporters ABCA3 and NPC1. J Lipid Res 2013, 54:2153-2165

12. Alpy F, Latchumanan VK, Kedinger V, Janoshazi A, Thiele C, Wendling C, Rio MC, Tomasetto C: Functional characterization of the MENTAL domain. J Biol Chem 2005, 280:17945-17952

13. Kishida T, Kostetskii I, Zhang Z, Martinez F, Liu P, Walkley SU, Dwyer NK, Blanchette-Mackie EJ, Radice GL, Strauss JF 3rd: Targeted mutation of the MLN64 START domain causes only modest alterations in cellular sterol metabolism. J Biol Chem 2004, 279: 19276-19285

14. Hölttä-Vuori M, Alpy F, Tanhuanpää K, Jokitalo E, Mutka A-L, Ikonen E: MLN64 is involved in actin-mediated dynamics of late endocytic organelles. Mol Biol Cell 2005, 16:3873-3886

15. Charman M, Kennedy BE, Osborne N, Karten B: MLN64 mediates egress of cholesterol from endosomes to mitochondria in the absence of functional Niemann-Pick Type C1 protein. J Lipid Res 2010, 51: $1023-1034$

16. Zhang M, Liu P, Dwyer NK, Christenson LK, Fujimoto T, Martinez F, Comly M, Hanover JA, Blanchette-Mackie EJ, Strauss JF 3rd: MLN64 mediates mobilization of lysosomal cholesterol to steroidogenic mitochondria. J Biol Chem 2002, 277:33300-33310

17. Vinatzer U, Dampier B, Streubel B, Pacher M, Seewald MJ, Stratowa C, Kaserer K, Schreiber M: Expression of HER2 and the coamplified genes GRB7 and MLN64 in human breast cancer: quantitative real-time reverse transcription-PCR as a diagnostic alternative to immunohistochemistry and fluorescence in situ hybridization. Clin Cancer Res 2005, 11:8348-8357

18. Rasband WS: ImageJ. Bethesda, MD, National Institutes of Health, 1997-2012

19. Bligh EG, Dyer WJ: A rapid method of total lipid extraction and purification. Can J Biochem Physiol 1959, 37:911-917

20. Holtta-Vuori M, Vainio S, Kauppi M, Van Eck M, Jokitalo E, Ikonen E: Endosomal actin remodeling by coronin-1A controls lipoprotein uptake and degradation in macrophages. Circ Res 2012, 110:450-455

21. Pimplikar SW, Ikonen E, Simons K: Basolateral protein transport in streptolysin O-permeabilized MDCK cells. J Cell Biol 1994, 125: $1025-1035$

22. Joensuu H, Isola J, Lundin M, Salminen T, Holli K, Kataja V, Pylkkänen L, Turpeenniemi-Hujanen T, von Smitten K, Lundin J: Amplification of erbB2 and erbB2 expression are superior to estrogen receptor status as risk factors for distant recurrence in pT1N0M0 breast cancer: a nationwide population-based study. Clin Cancer Res 2003, 9: 923-930

23. Joensuu H, Kellokumpu-Lehtinen P-L, Bono P, Alanko T, Kataja V, Asola R, Utriainen T, Kokko R, Hemminki A, Tarkkanen M, Turpeenniemi-Hujanen T, Jyrkkiö S, Flander M, Helle L, Ingalsuo S, Johansson K, Jääskeläinen A-S, Pajunen M, Rauhala M, KalevaKerola J, Salminen T, Leinonen M, Elomaa I, Isola J; FinHer Study Investigators: Adjuvant docetaxel or vinorelbine with or without trastuzumab for breast cancer. N Engl J Med 2006, 354:809-820

24. Joensuu H, Sperinde J, Leinonen M, Huang W, Weidler J, Bono P, Kataja V, Kokko R, Turpeenniemi-Hujanen T, Jyrkkio S, Isola J, Kellokumpu-Lehtinen PL, Paquet A, Lie Y, Bates M: Very high quantitative tumor HER2 content and outcome in early breast cancer. Ann Oncol 2011, 22:2007-2013

25. Yan D, Mäyränpää MI, Wong J, Perttilä J, Lehto M, Jauhiainen M, Kovanen PT, Ehnholm C, Brown AJ, Olkkonen VM: OSBP-related protein 8 (ORP8) suppresses ABCA1 expression and cholesterol efflux from macrophages. J Biol Chem 2008, 283:332-340

26. Sihto H, Kukko H, Koljonen V, Sankila R, Böhling T, Joensuu H: Clinical factors associated with Merkel cell polyomavirus infection in Merkel cell carcinoma. J Natl Cancer Inst 2009, 101:938-945

27. Leivonen S-K, Sahlberg KK, Mäkelä R, Due EU, Kallioniemi O, Børresen-Dale A-L, Perälä M: High-throughput screens identify microRNAs essential for HER2 positive breast cancer cell growth. Mol Oncol 2014, 8:93-104 
28. Linder MD, Uronen RL, Holtta-Vuori M, van der Sluijs P, Peranen J, Ikonen E: Rab8-dependent recycling promotes endosomal cholesterol removal in normal and sphingolipidosis cells. Mol Biol Cell 2007, 18: $47-56$

29. Ikonen E: Cellular cholesterol trafficking and compartmentalization. Nat Rev Mol Cell Biol 2008, 9:125-138

30. Bhakdi S, Tranum-Jensen J, Sziegoleit A: Mechanism of membrane damage by streptolysin-O. Infect Immun 1985, 47:52-60

31. Finn RS: Targeting Src in breast cancer. Ann Oncol 2008, 19: 1379-1386

32. Staubach S, Hanisch F-G: Lipid rafts: signaling and sorting platforms of cells and their roles in cancer. Expert Rev Proteomics 2011, 8: 263-277

33. Cai W, Ye L, Sun J, Mansel RE, Jiang WG: Expression of MLN64 influences cellular matrix adhesion of breast cancer cells, the role for focal adhesion kinase. Int J Mol Med 2010, 25:573-580

34. Alpy F, Boulay A, Moog-Lutz C, Andarawewa KL, Degot S, Stoll I, Rio M-C, Tomasetto C: Metastatic lymph node 64 (MLN64), a gene overexpressed in breast cancers, is regulated by $\mathrm{Sp} / \mathrm{KLF}$ transcription factors. Oncogene 2003, 22:3770-3780

35. Kauraniemi P, Bärlund M, Monni O, Kallioniemi A: New amplified and highly expressed genes discovered in the ERBB2 amplicon in breast cancer by cDNA microarrays. Cancer Res 2001, 61:8235-8240

36. Mohd Sharial MSN, Crown J, Hennessy BT: Overcoming resistance and restoring sensitivity to HER2-targeted therapies in breast cancer. Ann Oncol 2012, 23:3007-3016

37. Danilo C, Frank PG: Cholesterol and breast cancer development. Curr Opin Pharmacol 2012, 12:677-682
38. Silvente-Poirot S, Poirot M: Cancer: cholesterol and cancer, in the balance. Science 2014, 343:1445-1446

39. Simons K, Ikonen E: Functional rafts in cell membranes. Nature 1997, 387:569-572

40. Ramprasad OG, Srinivas G, Rao KS, Joshi P, Thiery JP, Dufour S, Pande G: Changes in cholesterol levels in the plasma membrane modulate cell signaling and regulate cell adhesion and migration on fibronectin. Cell Motil Cytoskeleton 2007, 64:199-216

41. Raghu H, Sodadasu PK, Malla RR, Gondi CS, Estes N, Rao JS: Localization of UPAR and MMP-9 in lipid rafts is critical for migration, invasion and angiogenesis in human breast cancer cells. BMC Cancer 2010, 10:647

42. Kanerva K, Uronen R-L, Blom T, Li S, Bittman R, Lappalainen P, Peränen J, Raposo G, Ikonen E: LDL cholesterol recycles to the plasma membrane via a Rab8a-Myosin5b-actin-dependent membrane transport route. Dev Cell 2013, 27:249-262

43. Patra SK: Dissecting lipid raft facilitated cell signaling pathways in cancer. Biochim Biophys Acta 2008, 1785:182-206

44. Heino S, Lusa S, Somerharju P, Ehnholm C, Olkkonen VM, Ikonen E: Dissecting the role of the golgi complex and lipid rafts in biosynthetic transport of cholesterol to the cell surface. Proc Natl Acad Sci U S A 2000, 97:8375-8380

45. Zhang S, Huang W-C, Li P, Guo H, Poh S-B, Brady SW, Xiong Y, Tseng L-M, Li S-H, Ding Z, Sahin AA, Esteva FJ, Hortobagyi GN, Yu D: Combating trastuzumab resistance by targeting SRC, a common node downstream of multiple resistance pathways. Nat Med 2011, 17:461-469

46. Vu T, Claret FX: Trastuzumab: updated mechanisms of action and resistance in breast cancer. Front Oncol 2012, 2:62 